Volume 70, Number 2, Pages 678689(2021)

DOI: $10.31801 /$ cfsuasmas.797257

ISSN 1303-5991 E-ISSN 2618-6470

Received by the editors: September 19, 2020; Accepted: March 11, 2021

\title{
SOLUTION OF FRACTIONAL KINETIC EQUATIONS INVOLVING GENERALIZED HURWITZ-LERCH ZETA FUNCTION USING SUMUDU TRANSFORM
}

\author{
Oğuz YAĞCI and Recep ŞAHİN \\ Department of Mathematics, Faculty of Arts and Sciences, Kırıkkale University,
} 71450, Kirıkkale, TURKEY

\begin{abstract}
Fractional kinetic equations (FKEs) comprising a large array of special functions have been extensively and successfully applied in specification and solving many significant problems of astrophysics and physics. In this present work, our aim is to demonstrate solutions of (FKEs) of the generalized Hurwitz-Lerch Zeta function by applying the Sumudu transform. In addition to these, solutions of (FKEs) in special conditions of generalised Hurwitz-Lerch Zeta function have been derived.
\end{abstract}

\section{Introduction}

The Hurwitz-Lerch Zeta function is defined by 34,35 :

$$
\Phi(\zeta, m, \alpha)=\sum_{n=0}^{\infty} \frac{\zeta^{n}}{(n+\alpha)^{m}}
$$

$$
\left(\alpha \in \mathbb{C} \backslash \mathbb{Z}_{0} ; m \in \mathbb{C} \text { when }|\zeta|<1 ; \Re(m)>1 \text { when }|\zeta|=1\right) .
$$

Many researchers studied many different generalisations and extensions of the Hurwitz-Lerch Zeta function by inserting certain additional parameters to the series representation of the Hurwitz-Lerch Zeta function. The interested readers can refer to these earlier publications for further researches and applications $13,14,15,18$,

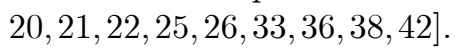

2020 Mathematics Subject Classification. Primary 11M35, 44A20, 33E12; Secondary 33B15, $26 \mathrm{~A} 33$.

Keywords and phrases. Hurwitz-Lerch Zeta function, generalized Hurwitz-Lerch Zeta function, fractional calculus, fractional kinetic equation, Mittag-Leffler function, Sumudu transform.

$\triangle$ 1588151031@kku.edu.tr, oguzyagci26@gmail.com- Corresponding author; recepsahin@kku.edu.tr

(D) 0000-0001-9902-8094; 0000-0001-5713-3830. 
In 2011, Srivastava et. al [41, p.491, Eq.(1.20)] introduced and studied the following extension of the generalized Hurwitz-Lerch Zeta function:

$$
\Phi_{\lambda, \mu ; \omega}^{(\sigma, \rho, \kappa)}(\zeta, m, a)=\sum_{n=0}^{\infty} \frac{(\lambda)_{\sigma n}(\mu)_{\rho n}}{(\omega)_{\kappa n} n !} \frac{\zeta^{n}}{(n+a)^{m}},
$$

$\left(\lambda, \mu \in \mathbb{C} ; a, \omega \in \mathbb{C} \backslash \mathbb{Z}_{0}^{-} ; \sigma, \rho, \kappa \in \mathbb{R}^{+} ; \kappa-\sigma-\rho>-1\right.$ when $m, \zeta \in \mathbb{C} ;$

$$
\begin{aligned}
& \kappa-\sigma-\rho=-1 \text { and } m \in \mathbb{C} \text { when }|\zeta|<\delta^{\star}=\sigma^{-\sigma} \rho^{-\rho} \kappa^{\kappa} ; \\
& \left.\kappa-\sigma-\rho=-1 \text { and } \Re(m+\omega-\lambda-\mu)>1 \text { when }|\zeta|=\delta^{\star}\right) .
\end{aligned}
$$

1.1. Fractional Kinetic Equations. In 23 one determinated the fractional differential equation for the rate of change of reaction. The destruction rate and the production rate follow:

$$
\frac{d \mathfrak{g}}{d \mathfrak{x}}=-\mathfrak{d}\left(\mathfrak{g}_{\mathfrak{x}}\right)+\mathfrak{p}\left(\mathfrak{g}_{\mathfrak{x}}\right),
$$

where $\mathfrak{g}=\mathfrak{g}(\mathfrak{x})$ the rate of the reaction, $\mathfrak{d}=\mathfrak{d}(\mathfrak{g})$ the rate of destruction, $\mathfrak{p}=\mathfrak{p}(\mathfrak{g})$ the rate of production and $\mathfrak{g}_{\mathfrak{x}}$ denotes the function defined by $\mathfrak{g}_{\mathfrak{x}}\left(\mathfrak{x}^{\star}\right)=\mathfrak{g}\left(\mathfrak{x}-\mathfrak{x}^{\star}\right), \mathfrak{x}^{\star}>0$.

The special condition of equation (3) for spatial fluctuations and inhomogeneities in $\mathfrak{g}(\mathfrak{x})$ the quantities are ignored, that is the equation

$$
\frac{d \mathfrak{g}}{d \mathfrak{x}}=-\mathfrak{c}_{i} \mathfrak{g}_{i}(\mathfrak{x})
$$

with the initial condition that $\mathfrak{g}_{i}(\mathfrak{x}=0)=\mathfrak{g}_{0}$ is the number of density of the species $i$ at time $\mathfrak{x}=0$ and $\mathfrak{c}_{i}>0$. If we shift the index $i$ and integrate the standard kinetic equation (4), we have

$$
\mathfrak{g}(\mathfrak{x})-\mathfrak{g}_{0}=-\mathfrak{c}_{o} \mathcal{D}_{t}^{-1} \mathfrak{g}(\mathfrak{x})
$$

where ${ }_{0} \mathcal{D}_{\mathfrak{x}}^{-1}$ is the special condition of the Riemann-Liouville integral operator ${ }_{0} \mathcal{D}_{\mathfrak{x}}^{-\xi}$ given as 40 ,

$$
\begin{aligned}
{ }_{0} \mathcal{D}_{\mathfrak{x}}^{-\xi} f(\mathfrak{x})= & \frac{1}{\Gamma(\xi)} \int_{0}^{\mathfrak{x}}(\mathfrak{x}-s)^{\xi-1} f(s) d s, \\
& (\mathfrak{x}>0, \Re(\xi)>0) .
\end{aligned}
$$

The fractional generalisation of the standard kinetic equation (5) is studied by Haubold and Mathai as follows 23]:

$$
\mathfrak{g}(\mathfrak{x})-\mathfrak{g}_{0}=-\mathfrak{c}^{\nu}{ }_{0} \mathcal{D}_{\mathfrak{x}}^{-1} \mathfrak{g}(\mathfrak{x})
$$

and acquired the solution of (4) as follows:

$$
\mathfrak{g}(\mathfrak{x})=\mathfrak{g}_{0} \sum_{k=0}^{\infty} \frac{(-1)^{k}}{\Gamma(\xi k+1)}(\mathfrak{c} \mathfrak{x})^{\xi k}
$$


In addition to that, Saxena and Kalla 30 take into consideration the following fractional kinetic equation:

$$
\mathfrak{g}(\mathfrak{x})-\mathfrak{g}_{0} f(\mathfrak{x})=-\mathfrak{c}^{\xi}{ }_{0} \mathcal{D}_{\mathfrak{x}}^{-1} \mathfrak{g}(\mathfrak{x})(\Re(\xi)>0),
$$

where $\mathfrak{g}(\mathfrak{x})$ denotes the number density of a given species at time $\mathfrak{x}, \mathfrak{g}_{0}=\mathfrak{g}(0)$ is the number of density of that species at time $\mathfrak{x}=0, \mathfrak{c}$ is a constant and $f \in L(0, \infty)$.

By taking advantage of the Laplace transform [19,37,39] to the equation (9),

$$
\begin{gathered}
\mathfrak{L}\{\mathfrak{g}(\mathfrak{x}) ; p\}=\mathfrak{g}_{0} \frac{F(p)}{1+\mathfrak{c}^{\nu} p^{-\nu}}=\mathfrak{g}_{0}\left(\sum_{n=0}^{\infty}\left(-\mathfrak{c}^{\nu}\right)^{n} p^{-\nu n}\right) F(p), \\
\left(n \in \mathfrak{g}_{0},\left|\frac{\mathfrak{c}}{p}\right|<1\right) .
\end{gathered}
$$

The extension and generalisation of (FKEs) comprising many fractional operators

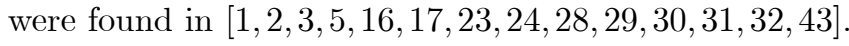

1.2. Sumudu Transform. The Sumudu transform is extensively used to solve several type of problems in science and engineering and it was introduced by Watagula 44, 45. For details, the reader is referred to [4,7,8, 9, 10, 11, 12]. is,

Suppose that $\mathcal{U}$ be the class of exponentially bounded function $f: \Re \rightarrow \Re$, that

$$
f(\zeta)< \begin{cases}\mathcal{M} \exp \left(-\frac{\zeta}{\eta_{1}}\right) & (\zeta \leqq 0) ; \\ \mathcal{M} \exp \left(\frac{\zeta}{\eta_{2}}\right) & (\zeta \geqq 0),\end{cases}
$$

where $\mathcal{M}, \eta_{1}$ and $\eta_{2}$ are positive real constants. The Sumudu transform defined on the set $\mathcal{U}$ is given as follows [44, 45]:

$$
\mathcal{G}(u)=\mathcal{S}\{f(\zeta) ; u\}=\int_{0}^{\infty} e^{-\zeta} f(u \zeta) d \zeta \quad\left(-\eta_{1}<u<\eta_{2}\right) .
$$

The main goal of this work is to demonstrate the generalized (FKEs) involving generalised Hurwitz-Lerch Zeta function (2). Here, we conceive the Sumudu transform methodology to arrive at the solutions.

\section{Main Results}

Here, we will explain the solution of the generalised (FKEs) which by considering generalized Hurwitz-Lerch Zeta function (2).

Theorem 1. If $\mathfrak{b}>0, \xi>0 ; \lambda, \mu, \delta \in \mathbb{C}$, and $\mathfrak{b} \neq \delta$ be such that $a, \omega \in$ $\mathbb{C} \backslash \mathbb{Z}_{0}^{-} ; \sigma, \rho, \kappa \in \mathbb{R}^{+}$, then the solution of the given fractional equation

$$
\mathfrak{g}(\mathfrak{x})-\mathfrak{g}_{0} \Phi_{\lambda, \mu ; \omega}^{(\sigma, \rho) \kappa)}\left(\mathfrak{b}^{\xi} \mathfrak{x}^{\nu}, m, a\right)=-\delta^{\xi}{ }_{0} \mathcal{D}_{\mathfrak{x}}^{-\xi} \mathfrak{g}(\mathfrak{x})
$$


FKE INVOLVING HURWITZ-LERCH ZETA FUNCTION USING SUMUDU TRANSFORM681

is derived by

$$
\mathfrak{g}(\mathfrak{x})=\mathfrak{g}_{0} \sum_{n=0}^{\infty} \frac{(\lambda)_{\sigma n}(\mu)_{\rho n} \Gamma(\xi n+1) \mathfrak{b}^{\xi n} t^{\xi n-1}}{(\omega)_{\kappa n} n !(n+a)^{m}} \mathcal{E}_{\xi, \xi n}\left(-\delta^{\xi} \mathfrak{x}^{\xi}\right)
$$

where $\mathcal{E}_{\xi, \xi n}($.$) is the Mittag-Leffler function [27].$

Proof. The Sumudu transform of the Riemann-Liouville fractional integral operator is defined by [24, p. 460, Eq. (2.10)]:

$$
\mathcal{S}\left[{ }_{0} \mathcal{D}_{\mathfrak{x}}^{-\xi} f(\mathfrak{x}) ; u\right]=\mathcal{S}\left[\frac{\mathfrak{x}^{\xi-1}}{\Gamma(\xi)} ; u\right] \cdot \mathcal{S}[f(\mathfrak{x}) ; u]=u^{\xi} G(u) .
$$

Now, taking advantage of the Sumudu transform to the both sides of 12 , we have

$$
\begin{aligned}
\mathcal{S}\{\mathfrak{g}(\mathfrak{x}) ; u\} & =\mathfrak{g}_{0} \mathcal{S}\left\{\Phi_{\lambda, \mu ; \omega}^{(\sigma, \rho, \kappa)}\left(\mathfrak{b}^{\nu} \mathfrak{x}^{\nu}, m, a\right) ; u\right\}-\delta^{\xi} \mathcal{S}\left\{{ }_{0} \mathcal{D}_{\mathfrak{x}}^{-\xi} \mathfrak{g}(\mathfrak{x}) ; u\right\} \\
\mathfrak{g}(u) & =\mathfrak{g}_{0}\left\{\int_{0}^{\infty} e^{-\mathfrak{x}} \sum_{n=0}^{\infty} \frac{(\lambda)_{\sigma n}(\mu)_{\rho n}\left(\mathfrak{b}^{\nu}(u \mathfrak{x})^{\xi}\right)^{n}}{(\omega)_{\kappa n} n !(n+a)^{m}}\right\} d \mathfrak{x}-\delta^{\xi} u^{\xi} \mathfrak{g}(u) \\
\mathfrak{g}(u)+\delta^{\xi} u^{\xi} \mathfrak{g}(u) & \\
= & \mathfrak{g}_{0} \sum_{n=0}^{\infty} \frac{(\lambda)_{\sigma n}(\mu)_{\rho n} \mathfrak{b}^{\xi n}}{(\omega)_{\kappa n} n !(n+a)^{m}} u^{\nu n} \int_{0}^{\infty} e^{-\mathfrak{x}} \mathfrak{x}^{\xi n} d \mathfrak{x} \\
& =\mathfrak{g}_{0} \sum_{n=0}^{\infty} \frac{(\lambda)_{\sigma n}(\mu)_{\rho n} \mathfrak{b}^{\xi n}}{(\omega)_{\kappa n} n !(n+a)^{m}} u^{\xi n} \Gamma(\xi n+1) \\
N(u) & =\mathfrak{g}_{0} \sum_{n=0}^{\infty} \frac{(\lambda)_{\sigma n}(\mu)_{\rho n} \Gamma(\xi n+1) \mathfrak{b}^{\xi n}}{(\omega)_{\kappa n} n !(n+a)^{m}} u^{\xi n} \sum_{r=0}^{\infty}\left[-(\delta u)^{\xi}\right]^{r} .
\end{aligned}
$$

Taking the inverse Sumudu transform of (15), and by applying

we have

$$
\mathcal{S}^{-1}\left\{u^{\xi} ; \mathfrak{x}\right\}=\frac{\mathfrak{x}^{\xi-1}}{\Gamma(\xi)},(\Re(\xi)>0),
$$

$$
\begin{aligned}
& \mathcal{S}^{-1}\{\mathfrak{g}(u)\}=\mathfrak{g}_{O} \sum_{n=0}^{\infty} \frac{(\lambda)_{\sigma n}(\mu)_{\rho n} \Gamma(\xi n+1) \mathfrak{b}^{\xi n}}{(\omega)_{\kappa n} n !(n+a)^{m}} \\
& \times \mathcal{S}^{-1}\left[\sum_{r=0}^{\infty} \delta^{\xi r} u^{\xi(n+r)}\right] \\
& \mathfrak{g}(\mathfrak{x})=\sum_{n=0}^{\infty} \frac{(\lambda)_{\sigma n}(\mu)_{\rho n} \Gamma(\xi n+1) \mathfrak{b}^{\xi n} \mathfrak{x}^{\xi n-1}}{(\omega)_{\kappa n} n !(n+a)^{m}} \sum_{r=0}^{\infty}(-1)^{r} \delta^{\xi r} \frac{\mathfrak{x}^{\xi r}}{\Gamma(\xi n+\xi r)} .
\end{aligned}
$$

So, we can be yield the required result (13). 
Theorem 2. If $\mathfrak{b}>0, \xi>0 ; \lambda, \mu \in \mathbb{C}$ be such that $a, \omega \in \mathbb{C} \backslash \mathbb{Z}_{0}^{-} ; \sigma, \rho, \kappa \in \mathbb{R}^{+}$, then the solution of the given fractional equation

$$
\mathfrak{g}(\mathfrak{x})-\mathfrak{g}_{0} \Phi_{\lambda, \mu ; \omega}^{(\sigma, \rho)}\left(\mathfrak{b}^{\xi} \mathfrak{x}^{\xi}, m, a\right)=-\mathfrak{b}^{\xi}{ }_{0} \mathcal{D}_{\mathfrak{x}}^{-\xi} \mathfrak{g}(\mathfrak{x})
$$

is derived by

$$
\mathfrak{g}(\mathfrak{x})=\mathfrak{g}_{0} \sum_{n=0}^{\infty} \frac{(\lambda)_{\sigma n}(\mu)_{\rho n} \Gamma(\xi n+1) \mathfrak{b}^{\xi n} \mathfrak{x}^{\xi n-1}}{(\omega)_{\kappa n} n !(n+a)^{m}} \mathcal{E}_{\xi, \xi n}\left(-\mathfrak{b}^{\xi} \mathfrak{x}^{\xi}\right),
$$

where $\mathcal{E}_{\xi, \xi n}($.$) is the Mittag-Leffler function [27].$

Proof. The proof of Theorem 2 is parallel to the proof of Theorem 1, thus the details are omitted.

Theorem 3. If $\xi>0 ; \lambda, \mu, \delta \in \mathbb{C}$ be such that $a, \omega \in \mathbb{C} \backslash \mathbb{Z}_{0}^{-} ; \sigma, \rho, \kappa \in \mathbb{R}^{+}$, then the solution of the given fractional equation

$$
\mathfrak{g}(\mathfrak{x})-\mathfrak{g}_{o} \Phi_{\lambda, \mu ; \omega}^{(\sigma, \rho, \kappa)}(\mathfrak{x}, m, a)=-\delta^{\xi}{ }_{o} \mathcal{D}_{\mathfrak{x}}^{-\xi} \mathfrak{g}(\mathfrak{x})
$$

is derived by

$$
\mathfrak{g}(\mathfrak{x})=\mathfrak{g}_{0} \sum_{n=0}^{\infty} \frac{(\lambda)_{\sigma n}(\mu)_{\rho n} \Gamma(n+1) \mathfrak{x}^{n-1}}{(\omega)_{\kappa n} n !(n+a)^{m}} \mathcal{E}_{\xi, n}\left(-\delta^{\xi} \mathfrak{x}^{\xi}\right),
$$

where $\mathcal{E}_{\xi, n}($.$) is the Mittag-Leffler function [27].$

Proof. Theorem 3 can be easily acquired from Theorem 1, so the details are omitted.

2.1. Special Conditions. Choosing $\lambda=\sigma=1$ in the equation (2), which is the generalized Hurwitz-Lerch Zeta function $\Phi_{\mu ; \omega}^{\rho, \kappa}(\zeta, m, a)$ introduced and studied by Lin and Srivastava 25].

Applying $\lambda=\sigma=1$ in the Theorem 1, Theorem 2, Theorem 3 obtained the following forms:

Corollary 4. If $\mathfrak{b}>0, \xi>0 ; \mu, \delta \in \mathbb{C}$, and $\mathfrak{b} \neq \delta$ be such that $a, \omega \in \mathbb{C} \backslash$ $\mathbb{Z}_{0}^{-} ; \rho, \kappa \in \mathbb{R}^{+}$, then the solution of the given fractional equation

$$
\mathfrak{g}(\mathfrak{x})-\mathfrak{g}_{0} \Phi_{\mu ; \omega}^{(\rho, \kappa)}\left(\mathfrak{b}^{\xi} \mathfrak{x}^{\xi}, m, a\right)=-\delta^{\xi}{ }_{0} \mathcal{D}_{\mathfrak{x}}^{-\xi} \mathfrak{g}(\mathfrak{x})
$$

is derived by

$$
\mathfrak{g}(\mathfrak{x})=\mathfrak{g}_{0} \sum_{n=0}^{\infty} \frac{(\mu)_{\rho n} \Gamma(\xi n+1) \mathfrak{b}^{\xi n} \mathfrak{x}^{\xi n-1}}{(\omega)_{\kappa n}(n+a)^{m}} \mathcal{E}_{\xi, \xi n}\left(-\delta^{\xi} \mathfrak{x}^{\xi}\right) .
$$


FKE INVOLVING HURWITZ-LERCH ZETA FUNCTION USING SUMUDU TRANSFORM683

Corollary 5. If $\mathfrak{b}>0, \xi>0 ; \mu \in \mathbb{C}$ be such that $a, \omega \in \mathbb{C} \backslash \mathbb{Z}_{0}^{-} ; \rho, \kappa \in \mathbb{R}^{+}$, then the solution of the given fractional equation

$$
\mathfrak{g}(\mathfrak{x})-\mathfrak{g}_{0} \Phi_{\mu ; \omega}^{(\rho, \kappa)}\left(\mathfrak{b}^{\xi} \mathfrak{x}^{\xi}, m, a\right)=-\mathfrak{b}^{\xi}{ }_{o} \mathcal{D}_{\mathfrak{x}}^{-\xi} \mathfrak{g}(\mathfrak{x})
$$

is derived by

$$
\mathfrak{g}(\mathfrak{x})=\mathfrak{g}_{0} \sum_{n=0}^{\infty} \frac{(\mu)_{\rho n} \Gamma(\xi n+1) \mathfrak{b}^{\xi n} \mathfrak{x}^{\xi n-1}}{(\omega)_{\kappa n}(n+a)^{m}} \mathcal{E}_{\xi, \xi n}\left(-\mathfrak{b}^{\nu} \mathfrak{x}^{\xi}\right) .
$$

Corollary 6. If $\mu, \delta \in \mathbb{C}$ be such that $a, \omega \in \mathbb{C} \backslash \mathbb{Z}_{0}^{-} ; \rho, \kappa \in \mathbb{R}^{+}$, then the solution of the given fractional equation

$$
\mathfrak{g}(\mathfrak{x})-\mathfrak{g}_{o} \Phi_{\mu ; \omega}^{(\rho, \kappa)}(\mathfrak{x}, m, a)=-\delta^{\xi}{ }_{o} \mathcal{D}_{\mathfrak{x}}^{-\xi} \mathfrak{g}(\mathfrak{x})
$$

is derived by

$$
\mathfrak{g}(\mathfrak{x})=\mathfrak{g}_{0} \sum_{n=0}^{\infty} \frac{(\mu)_{\rho n} \Gamma(n+1) \mathfrak{x}^{n-1}}{(\omega)_{\kappa n}(n+a)^{m}} \mathcal{E}_{\xi, n}\left(-\delta^{\xi} \mathfrak{x}^{\xi}\right) .
$$

Setting $\sigma=\rho=\kappa=1$ in the equation (2), which is the generalized HurwitzLerch Zeta function $\Phi_{\lambda, \mu ; \omega}(\zeta, m, a)$ introduced and studied by Garg et. all [20].

Applying $\sigma=\rho=\kappa=1$ in the Theorem 1, Theorem 2, Theorem 3 obtained the following forms:

Corollary 7. If $\mathfrak{b}>0, \xi>0 ; \lambda, \mu, \delta \in \mathbb{C}$, and $\mathfrak{b} \neq \delta$ be such that $a, \omega \in \mathbb{C} \backslash \mathbb{Z}_{0}^{-}$, then the solution of the following given equation

$$
\mathfrak{g}(\mathfrak{x})-\mathfrak{g}_{O} \Phi_{\lambda, \mu ; \omega}\left(\mathfrak{b}^{\xi} \mathfrak{x}^{\xi}, m, a\right)=-\delta^{\xi}{ }_{0} \mathcal{D}_{\mathfrak{x}}^{-\xi} \mathfrak{g}(\mathfrak{x})
$$

is derived by

$$
\mathfrak{g}(\mathfrak{x})=\mathfrak{g}_{0} \sum_{n=0}^{\infty} \frac{(\lambda)_{n}(\mu)_{n} \Gamma(\xi n+1) \mathfrak{b}^{\xi n} \mathfrak{x}^{\xi n-1}}{(\omega)_{n} n !(n+a)^{m}} \mathcal{E}_{\xi, \xi n}\left(-\delta^{\xi} \mathfrak{x}^{\xi}\right) .
$$

Corollary 8. If $\mathfrak{b}>0, \xi>0 ; \lambda, \mu \in \mathbb{C}$ be such that $a, \omega \in \mathbb{C} \backslash \mathbb{Z}_{0}^{-}$, then the solution of the given fractional equation

$$
\mathfrak{g}(\mathfrak{x})-\mathfrak{g}_{0} \Phi_{\lambda, \mu ; \omega}\left(\mathfrak{b}^{\xi} \mathfrak{x}^{\xi}, m, a\right)=-\mathfrak{b}^{\xi}{ }_{0} \mathcal{D}_{\mathfrak{x}}^{-\xi} \mathfrak{g}(\mathfrak{x})
$$

is derived by

$$
\mathfrak{g}(\mathfrak{x})=\mathfrak{g}_{0} \sum_{n=0}^{\infty} \frac{(\lambda)_{n}(\mu)_{n} \Gamma(\xi n+1) \mathfrak{b}^{\xi n} \mathfrak{x}^{\xi n-1}}{(\omega)_{n} n !(n+a)^{m}} \mathcal{E}_{\xi, \xi n}\left(-\mathfrak{b}^{\xi} \mathfrak{x}^{\xi}\right) .
$$

Corollary 9. If $\lambda, \mu, \delta \in \mathbb{C}$ be such that $a, \omega \in \mathbb{C} \backslash \mathbb{Z}_{0}^{-}$, then the solution of the given fractional equation

$$
\mathfrak{g}(\mathfrak{x})-\mathfrak{g}_{0} \Phi_{\lambda, \mu ; \omega}(\mathfrak{x}, m, a)=-\delta^{\xi}{ }_{0} \mathcal{D}_{\mathfrak{x}}^{-\xi} \mathfrak{g}(\mathfrak{x})
$$


is derived by

$$
\mathfrak{g}(\mathfrak{x})=\mathfrak{g}_{0} \sum_{n=0}^{\infty} \frac{(\lambda)_{n}(\mu)_{n} \Gamma(n+1) \mathfrak{x}^{n-1}}{(\omega)_{n} n !(n+a)^{m}} \mathcal{E}_{\xi, n}\left(-\delta^{\xi} \mathfrak{x}^{\xi}\right) .
$$

Upon taking $\sigma=\rho=\kappa=1$ and $\lambda=\omega$ in the equation (2), which is the generalized Hurwitz-Lerch Zeta function $\Phi_{\mu}^{\star}(\zeta, m, a)$ introduced and studied by Goyal and Laddha [21, p.100, Eq.(1.5)].

Applying $\sigma=\rho=\kappa=1$ and $\lambda=\omega$ in the Theorem 1, Theorem 2, Theorem 3 obtained the following forms:

Corollary 10. If $\mathfrak{b}>0, \xi>0 ; \mu, \delta \in \mathbb{C}$, and $\mathfrak{b} \neq \delta$ be such that $a \in \mathbb{C} \backslash \mathbb{Z}_{0}^{-}$, then the solution of the given fractional equation

$$
\mathfrak{g}(\mathfrak{x})-\mathfrak{g}_{0} \Phi_{\mu}^{\star}\left(\mathfrak{b}^{\xi} \mathfrak{x}^{\xi}, m, a\right)=-\delta^{\xi}{ }_{o} \mathcal{D}_{\mathfrak{x}}^{-\xi} \mathfrak{g}(\mathfrak{x})
$$

is derived by

$$
\mathfrak{g}(\mathfrak{x})=\mathfrak{g}_{0} \sum_{n=0}^{\infty} \frac{(\mu)_{n} \Gamma(\xi n+1) \mathfrak{b}^{\xi n} \mathfrak{x}^{\xi n-1}}{n !(n+a)^{m}} \mathcal{E}_{\xi, \xi n}\left(-\delta^{\xi} \mathfrak{x}^{\xi}\right) .
$$

Corollary 11. If $\mathfrak{b}>0, \xi>0 ; \mu \in \mathbb{C}$ be such that $a \in \mathbb{C} \backslash \mathbb{Z}_{0}^{-}$, then the solution of the given fractional equation

$$
\mathfrak{g}(\mathfrak{x})-\mathfrak{g}_{0} \Phi_{\mu}^{\star}\left(\mathfrak{b}^{\xi} \mathfrak{x}^{\xi}, m, a\right)=-\mathfrak{b}^{\xi}{ }_{o} \mathcal{D}_{\mathfrak{x}}^{-\xi} \mathfrak{g}(\mathfrak{x})
$$

is derived by

$$
\mathfrak{g}(\mathfrak{x})=\mathfrak{g}_{0} \sum_{n=0}^{\infty} \frac{(\mu)_{n} \Gamma(\xi n+1) \mathfrak{b}^{\xi n} \mathfrak{x}^{\xi n-1}}{n !(n+a)^{m}} \mathcal{E}_{\xi, \xi n}\left(-\mathfrak{b}^{\xi} \mathfrak{x}^{\xi}\right) .
$$

Corollary 12. If $\lambda, \mu, \delta \in \mathbb{C}$ be such that $a \in \mathbb{C} \backslash \mathbb{Z}_{0}^{-}$, then the solution of the given fractional equation

$$
\mathfrak{g}(\mathfrak{x})-\mathfrak{g}_{0} \Phi_{\mu}^{\star}(\mathfrak{x}, m, a)=-\delta^{\xi}{ }_{0} \mathcal{D}_{\mathfrak{x}}^{-\xi} \mathfrak{g}(\mathfrak{x})
$$

is derived by

$$
\mathfrak{g}(\mathfrak{x})=\mathfrak{g}_{0} \sum_{n=0}^{\infty} \frac{(\mu)_{n} \Gamma(n+1) \mathfrak{x}^{n-1}}{n !(n+a)^{m}} \mathcal{E}_{\xi, n}\left(-\delta^{\xi} \mathfrak{x}^{\xi}\right)
$$

Upon taking $\sigma=\rho=\mu=1$ and $\zeta=\frac{\zeta}{\lambda}$. Then, the limit case of (2) when $\lambda \rightarrow \infty$, would yield the Mittag-Leffler type function $\mathcal{E}_{\kappa, \omega}^{(a)}(m ; \mathfrak{x})$ studied by Barnes 6], that is,

$$
\begin{array}{r}
\mathcal{E}_{\kappa, \omega}^{(a)}(m ; \zeta)=\sum_{n=0}^{\infty} \frac{\zeta^{n}}{(n+a)^{m} \Gamma(\omega+\kappa n)}, \\
\left(a, \omega \in \mathbb{C} \backslash \mathbb{Z}_{0}^{-} ; \Re(\kappa)>0 ; m, \zeta \in \mathbb{C}\right) .
\end{array}
$$

Applying $\sigma=\rho=\mu=1$ and $\zeta=\frac{\zeta}{\lambda}$. Then, the limit case of 2 when $\lambda \rightarrow \infty$ in the Theorem 1, Theorem 2, Theorem 3 obtained the following forms: 
FKE INVOLVING HURWITZ-LERCH ZETA FUNCTION USING SUMUDU TRANSFORM685

Corollary 13. If $\mathfrak{b}>0, \xi>0 ; \kappa, \delta \in \mathbb{C}$, and $\mathfrak{b} \neq \delta$ be such that $a, \omega \in \mathbb{C} \backslash \mathbb{Z}_{0}^{-}$, then the solution of the given fractional equation

$$
\mathfrak{g}(\mathfrak{x})-\mathfrak{g}_{0} \mathcal{E}_{\kappa, \omega}^{(a)}\left(m ; \mathfrak{b}^{\xi} \mathfrak{x}^{\xi}\right)=-\delta^{\xi}{ }_{o} \mathcal{D}_{\mathfrak{x}}^{-\xi} \mathfrak{g}(\mathfrak{x})
$$

is derived by

$$
\mathfrak{g}(\mathfrak{x})=\mathfrak{g}_{0} \sum_{n=0}^{\infty} \frac{\Gamma(\xi n+1) \mathfrak{b}^{\xi n} \mathfrak{x}^{\xi n-1}}{(n+a)^{m} \Gamma(\omega+\kappa n)} \mathcal{E}_{\xi, \xi n}\left(-\delta^{\xi} \mathfrak{x}^{\xi}\right) .
$$

Corollary 14. If $\mathfrak{b}>0, \xi>0 ; \kappa \in \mathbb{C}$ be such that $a, \omega \in \mathbb{C} \backslash \mathbb{Z}_{0}^{-}$, then the solution of the given fractional equation

$$
\mathfrak{g}(\mathfrak{x})-\mathfrak{g}_{0} \mathcal{E}_{\kappa, \omega}^{(a)}\left(m ; \mathfrak{b}^{\xi} \mathfrak{x}^{\xi}\right)=-\mathfrak{b}^{\xi}{ }_{0} \mathcal{D}_{\mathfrak{x}}^{-\xi} \mathfrak{g}(\mathfrak{x})
$$

is derived by

$$
\mathfrak{g}(\mathfrak{x})=\mathfrak{g}_{0} \sum_{n=0}^{\infty} \frac{\Gamma(\xi n+1) \mathfrak{b}^{\xi n} \mathfrak{x}^{\xi n-1}}{(n+a)^{m} \Gamma(\omega+\kappa n)} \mathcal{E}_{\xi, \xi n}\left(-\mathfrak{b}^{\xi} \mathfrak{x}^{\xi}\right) .
$$

Corollary 15. If $\kappa, \delta \in \mathbb{C}$ be such that $a, \omega \in \mathbb{C} \backslash \mathbb{Z}_{0}^{-}$, then the solution of the given fractional equation

$$
\mathfrak{g}(\mathfrak{x})-\mathfrak{g}_{0} \mathcal{E}_{\kappa, \omega}^{(a)}(m ; \mathfrak{x})=-\delta^{\xi}{ }_{o} \mathcal{D}_{\mathfrak{x}}^{-\xi} \mathfrak{g}(\mathfrak{x})
$$

is derived by

$$
\mathfrak{g}(\mathfrak{x})=\mathfrak{g}_{0} \sum_{n=0}^{\infty} \frac{\Gamma(n+1) \mathfrak{x}^{n-1}}{(n+a)^{m} \Gamma(\omega+\kappa n)} \mathcal{E}_{\xi, n}\left(-\delta^{\xi} \mathfrak{x}^{\xi}\right) .
$$

Finally, upon setting $\lambda, \mu, \omega, \sigma, \rho, \kappa=1$ in the equation (2), which gives the equation (1) 34, 35.

Choosing $\lambda, \mu, \omega, \sigma, \rho, \kappa=1$ in the Theorem 1, Theorem 2, Theorem 3 obtained the following forms:

Corollary 16. If $\mathfrak{b}>0 ; \delta, \xi \in \mathbb{C}, a \in \mathbb{C} \backslash \mathbb{Z}_{0}^{-}$, and $\mathfrak{b} \neq \delta$, then the solution of the given fractional equation

$$
\mathfrak{g}(\mathfrak{x})-\mathfrak{g}_{o} \Phi\left(\mathfrak{b}^{\xi} \mathfrak{x}^{\xi}, m, a\right)=-\delta^{\xi}{ }_{0} \mathcal{D}_{\mathfrak{x}}^{-\xi} \mathfrak{g}(\mathfrak{x})
$$

is derived by

$$
\mathfrak{g}(\mathfrak{x})=\mathfrak{g}_{0} \sum_{n=0}^{\infty} \frac{\Gamma(\xi n+1) \mathfrak{b}^{\xi n} \mathfrak{x}^{\xi n-1}}{(n+a)^{m}} \mathcal{E}_{\xi, \xi n}\left(-\delta^{\xi} \mathfrak{x}^{\xi}\right) .
$$

Corollary 17. If $\mathfrak{b}>0 ; \xi \in \mathbb{C}, a \in \mathbb{C} \backslash \mathbb{Z}_{0}^{-}$, then the solution of the given fractional equation

$$
\mathfrak{g}(\mathfrak{x})-\mathfrak{g}_{0} \Phi\left(\mathfrak{b}^{\xi} \mathfrak{x}^{\xi}, m, a\right)=-\mathfrak{b}^{\xi}{ }_{0} \mathcal{D}_{\mathfrak{x}}^{-\xi} \mathfrak{g}(\mathfrak{x})
$$


is derived by

$$
\mathfrak{g}(\mathfrak{x})=\mathfrak{g}_{0} \sum_{n=0}^{\infty} \frac{\Gamma(\xi n+1) \mathfrak{b}^{\xi n} \mathfrak{x}^{\xi n-1}}{(n+a)^{m}} \mathcal{E}_{\xi, \xi n}\left(-\mathfrak{b}^{\xi} \mathfrak{x}^{\xi}\right) .
$$

Corollary 18. If $\delta \in \mathbb{C}, a \in \mathbb{C} \backslash \mathbb{Z}_{0}^{-}$, then the solution of the given fractional equation

$$
\mathfrak{g}(\mathfrak{x})-\mathfrak{g}_{0} \Phi(\mathfrak{x}, m, a)=-\delta^{\xi}{ }_{0} \mathcal{D}_{\mathfrak{x}}^{-\xi} \mathfrak{g}(\mathfrak{x})
$$

is derived by

$$
\mathfrak{g}(\mathfrak{x})=\mathfrak{g}_{0} \sum_{n=0}^{\infty} \frac{\Gamma(n+1) \mathfrak{x}^{n-1}}{(n+a)^{m}} \mathcal{E}_{\xi, n}\left(-\delta^{\xi} \mathfrak{x}^{\xi}\right) .
$$

\section{Numerical Result and Graphic}

In this section, we present the $2 \mathrm{D}$ plots of Equation 13 for special values such as: $\lambda, \mu, \omega, \rho, \kappa, \sigma, a, m=1, \delta=4, \mathfrak{g}_{0}=3$ and $\xi=0.4,0.5,0.6$.

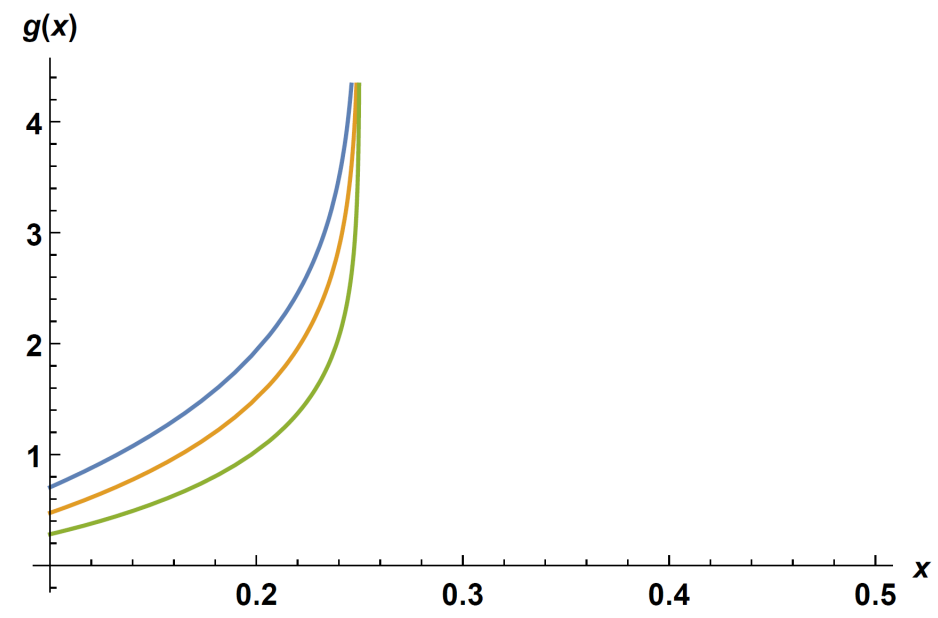

Figure 1. Solution of the FKE for GHLZ

\section{Conclusions}

The fractional kinetic equation involving the generalized Hurwitz-Lerch Zeta function is studied using the Sumudu transform. The results obtained in this study have remarkable significance as the solution of the equations are general and can be reproduced many new and known solutions of (FKEs) involving various type of special functions. 
Authors Contribution Statement All authors have contributed sufficiently in the planning, execution, or analysis of this study to be included as authors. All authors read and approved the final manuscript

Declaration of Competing Interests The authors declare that they have no known competing financial interest or personal relationships that could have appeared to influence the work reported in this paper.

Acknowledgements The authors are thankful to the reviewers and the editors for helpful suggestions which lead to essential improvement of the manuscript

\section{REFERENCES}

[1] Agarwal, G., Nisar, K. S., Certain fractional kinetic equations involving generalized Kfunctions, Analysis, 39(2) (2019), 65-70. https://doi.org/10.1515/anly-2019-0013

[2] Agarwal, P., Chand, M., Singh, G., Certain fractional kinetic equations involving the product of generalized k-Bessel function, Alexandria Engineering Journal, 55(4) (2016), 3053-3059. https://doi.org/10.1016/j.aej.2016.07.025

[3] Agarwal, P., Ntouyas, S. K., Jain, S., Chand, M., Singh, G., Fractional kinetic equations involving generalized k-Bessel function via Sumudu transform, Alexandria engineering journal, 57(3) (2018), 1937-1942. https://doi.org/10.1016/j.aej.2017.03.046

[4] Asiru, M. A., Sumudu transform and the solution of integral equation of convolution type, Int. J. Math. Educ. Sci. Technol., 32 (2001), 906-910. https://doi.org/10.1080/002073901317147870

[5] Baleanu, D., Khan, O., Khan, N., Nisar, K. S., Computable solution of fractional kinetic equations using Mathieu-type series, Advances in Difference Equations, 2019(1) (2019), 1-13. https://doi.org/10.1186/s13662-019-2167-4

[6] Barnes E. W., The asymptotic expansion of integral functions defined by Taylor series, Philos. Trans. Roy. Soc. London. Ser. A, 206 (1906), 249-297.

[7] Belgacem, F. B. M., Introducing and analyzing deeper Sumudu properties, Nonlinear Stud., 13 (2006), 23-42.

[8] Belgacem, F. B. M., Sumudu applications to Maxwells equations, PIERS Online, 5 (2009), 355-360. https://doi.org/10.2529/PIERS090120050621

[9] Belgacem, F. B. M., Applications with the Sumudu transform to Bessel functions and equations, Appl. Math. Sci., 4 (2010), 3665-3686.

[10] Belgacem, F. B. M., Al-Shemas, E. H., Silambarasan, R., Sumudu computation of the transient magnetic field in a lossy medium, Appl. Math. Inf. Sci., 6 (2016), 1-9.

[11] Belgacem, F. B. M., Karaballi, A. A., Sumudu transform fundamental properties investigations and applications, J. Appl. Math. Stoch. Anal., 2006 (2006), 1-23.

[12] Belgacem, F. B. M., Karaballi, A. A., Kalla, S. L., Analytical investigations of the Sumudu transform and applications to integral production equations, Math. Probl. Eng., 3 (2003), 103-118.

[13] Choi, J., Jang, D. S., Srivastava, H. M., A generalization of the HurwitzLerch Zeta function, Integral Transforms Spec. Funct., 19 (2008), 65-79. https://doi.org/10.1080/10652460701528909

[14] Choi, J., Parmar, R. K., An extension of the generalized Hurwitz-Lerch Zeta function of two variables, Filomat, 31 (2017), 91-96. https://doi.org/10.2298/FIL1701091C

[15] Choi, J., Şahin, R., Yağcı, O., Kim, D., Note on the Hurwitz-Lerch Zeta function of two variables, Symmetry, 12(9) (2020), 1431. https://doi.org/10.3390/sym12091431 
[16] Chouhan, A., Sarswat, S., On solution of generalized Kinetic equation of fractional order, Int. Jr. of Mathematical Sciences and Applications, 2(2) (2012), 813-818.

[17] Chouhan, A., Purohit, S. D., Sarswat, S., An alternative method for solving generalized differential equations of fractional order, Kragujevac J Math, 37 (2013), 299-306.

[18] Daman, O., Pathan, M. A., A further generalization of the Hurwitz Zeta function, Math. Sci. Res. J., 16(10) (2012), 251-259.

[19] Erdélyi, A., Magnus, W., Oberhettinger, F., Tricomi, F. G., Higher Transcendental Functions, Vol. I, McGraw-Hill Book Company, New York, Toronto and London, 1953. https://doi.org/10.1002/zamm.19540341220

[20] Garg, M., Jain, K., Kalla, S. L., A further study of general Hurwitz-Lerch zeta function, Algebras Groups Geom., 25 (2008), 311-319.

[21] Goyal, S. P., Laddha, R. K., On the generalized Zeta function and the generalized Lambert function, Ganita Sandesh, 11 (1997), 99-108.

[22] Gupta, P. L., Gupta, R. C., Ong, S.-H., Srivastava, H. M., A class of Hurwitz-Lerch Zeta distributions and their applications in reliability, Appl. Math. Comput., 196 (2008), 521-531. https://doi.org/10.1016/j.amc.2007.06.012

[23] Haubold, H. J., Mathai, A. M., The fractional kinetic equation and thermonuclear functions, Astrophys. Space Sci., 327 (2000), 53-63. https://doi.org/10.1023/A:1002695807970

[24] Kumar, D., Choi, J., Srivastava, H. M., Solution of a general family of fractional kinetic equations associated with the generalized Mittag-Leffler function, Nonlinear Funct. Anal. Appl, 23(3) (2018), 455-471.

[25] Lin, S. D., Srivastava, H. M., Some families of the Hurwitz-Lerch Zeta functions and associated fractional derivative and other integral representations, Appl. Math. Comput., 154 (2004), 725-733. https://doi.org/10.1016/S0096-3003(03)00746-X

[26] Lin, S. D., Srivastava, H. M., Wang, P. Y., Some expansion formulas for a class of generalized Hurwitz-Lerch Zeta functions, Integral Transforms Spec. Funct., 17 (2006), 817-822. https://doi.org/10.1080/10652460600926923

[27] Mittag-Leffler, G. M., Sur la representation analytique d'une branche uniforme d'une fonction monogene, Acta. Math., 29 (1905), 101-181. https://doi.org/10.1007/BF02403200

[28] Nisar, K. S., Shaikh, A., Rahman, G., Kumar, D., Solution of fractional kinetic equations involving class of functions and Sumudu transform, Advances in Difference Equations, 2020(1) (2020), 1-11. https://doi.org/10.1186/s13662-020-2513-6

[29] Nisar, K. S., Generalized Mittag-Leffler Type Function: Fractional Integrations and Application to Fractional Kinetic Equations, Frontiers in Physics, 8 (2020), 33. https://doi.org/10.3389/fphy.2020.00033

[30] Saxena, R. K., Kalla, S. L., On the solutions of certain fractional kinetic equations, Applied Mathematics and Computation, 199(2) (2008), 504-511. https://doi.org/10.1016/j.amc.2007.10.005

[31] Saxena, R. K., Mathai, A. M., Haubold, H. J., On fractional kinetic equations, Astrophysics and Space Science, 282(1) (2002), 281-287. https://doi.org/10.1023/A:1021175108964

[32] Saxena, R. K., Mathai, A. M., Haubold, H. J., On generalized fractional kinetic equations, Physica A: Statistical Mechanics and its Applications, 344(3-4) (2004), 657-664. https://doi.org/10.1016/j.physa.2004.06.048

[33] Srivastava, H. M., A new family of the $\lambda$-generalized Hurwitz-Lerch Zeta functions with applications, Appl. Math. Inform. Sci., 8 (2014), 1485-1500. https://doi.org/10.12785/amis/080402

[34] Srivastava, H. M., Choi, J., Series Associated with the Zeta and Related Functions, Kluwer Acedemic Publishers, Dordrecht, Boston and London, 2001.

[35] Srivastava, H. M., Choi, J., Zeta and $q$-Zeta Functions and Associated Series and Integrals, Elsevier Science Publishers, Amsterdam, London and New York, 2012. 
FKE INVOLVING HURWITZ-LERCH ZETA FUNCTION USING SUMUDU TRANSFORM 689

[36] Srivastava, H. M., Jankov, D., Pogány, T. K., Saxena, R. K., Two-sided inequalities for the extended Hurwitz-Lerch Zeta function, Comput. Math. Appl., 62 (2011), 516-522. https://doi.org/10.1016/j.camwa.2011.05.035

[37] Srivastava, H. M., Karlsson, P. W., Multiple Gaussian Hypergeometric Series, Halsted Press (Ellis Horwood Limited, Chichester), John Wiley and Sons, New York, Chichester, Brisbane and Toronto, 1985.

[38] Srivastava, H. M., Luo, M. J., Raina, R. K., New results involving a class of generalized Hurwitz-Lerch Zeta functions and their applications, Turkish J. Anal. Number Theory, 1 (2013), 26-35.

[39] Srivastava, H. M., Manocha, H. L., A Treatise on Generating Functions, Halsted Press (Ellis Horwood Limited, Chichester), John Wiley and Sons, New York, Chichester, Brisbane and Toronto, 1984.

[40] Srivastava, H. M., Saxena, R. K., Operators of fractional integration and their applications, Applied Mathematics and Computation, 118(1) (2001), 1-52. https://doi.org/10.1016/S00963003(99)00208-8

[41] Srivastava, H. M., Saxena, R. K., Pogány, T. K., Saxena, R., Integral and computational representations of the extended Hurwitz-Lerch Zeta function, Integral Transforms Spec. Funct., 22 (2011), 487-506. https://doi.org/10.1080/10652469.2010.530128

[42] Srivastava, H. M., Şahin, R., Yağc1, O., A family of incomplete Hurwitz-Lerch zeta functions of two variable, Miskolc Mathematical Notes, 21(1) (2020), 401-415. https://doi.org/10.18514/MMN.2020.3058

[43] Şahin, R., Yağc1, O., Fractional calculus of the extended hypergeometric function, Applied Mathematics and Nonlinear Sciences, 5(1) (2020), 369-384. https://doi.org/10.2478/amns.2020.1.00035

[44] Watugala, G. K., Sumudu Transform-an integral transform to solve differential equations and control engineering problems, Inter.J. Math. Ed. Sci. Tech., 24 (1993), 35-42. https://doi.org/10.1080/0020739930240105

[45] Watugala, G. K., Sumudu Transform-a new integral transform to solve differential equations and control engineering problems, Mathematical Engineering in Industry, 6(4) (1998), 319329 . 